\title{
PERCEPTUAL MECHANISMS FOR REPLACING OLD WORDS AND ADAPTING NEW WORDS: ESTABLISHING A CROSS- TALK BETWEEN WORD REPLACEMENT AND CODE- SWITCHING IN ODIA
}

\author{
Shashikanta Tarai \\ Department of Humanities and Social Sciences, NIT Raipur, India
}

Email: starai.eng@nitrr.ac.in

\begin{abstract}
This paper presents an investigation on how a decrease in perception determines the replacement of underuse old words, and the increase of perception manifests the adaptation of overuse new words. A relationship between lexical replacement and code-switching is established by examining the perceptual mechanisms of lexical adaptation in Odia. To test lexical adaptation in terms of perceptual rate, a psycholinguistic experiment on old Sanskrit tatsam and their semanticequivalent English-borrowed words was conducted by using semantic-priming paradigm. Target words that were associated either positively and negatively with preceding Odia and English priming-words were presented in Odia orthography. Odia-English bilingual speakers were asked to comprehend the positive and negative association between target and priming words, and to respond by pressing left arrow-key for positive and right arrow-key for negative meaning. Measuring perceptual facilitation for adaptation and code-switching in terms of response time (ms), results show that disyllabic English-borrowed words are processed faster than their equivalent disyllabic Sanskrittatsam words. English negative priming words facilitate the processing of disyllabic Sanskrit-tatsam words, suggesting faster code-switching effect from English to Odia. Odia-English bilingual speakers have a tendency to adapt English disyllabic words in the place disyllabic Sanskrit-tatsam words, preserving the minimal disyllabic word structure of Odia.
\end{abstract}

Keywords: Lexical replacement; adaptation; borrowed words; semantic paradigm

\section{Introduction}

In language contact situation, the code-mixed words are introduced by bi/multilingual speakers to native monolingual speakers (Thomason, 2001; Thomason and Kaufman, 1988; Haspelmath, 2008). Switching between two or more languages within a single utterance provides insight into the specific character of the lexical replacement (Clyne, 2003; Romaine, 2006). Following the mechanism of lexical replacement through code-switching, most of the monolingual speakers use the English words while speaking their mother tongue in this globalized world (Romaine, 2006). Externally, the words get incorporated into their mother tongue (Calabrese and Wetzels, 2009). For example, most of the rural Indian speakers who do not have any formal education in English use several English words while speaking in their mother tongue (Patnaik and Pandit, 1986). Such words which become a part and parcel of the borrowing language are classified as English loanwords (Chaudhary, 2009). The adoption and use of words other than those of the native language expands the functional domain of the native language. Lexical borrowing and adaptation of loanwords enriches the vocabulary and expands the use of the native borrowing language. In the process of functional expansion, the borrowing language replaces some existing indigenous words with new borrowed words. Lexical enrichment and replacement through code switching are two ways in which a language updates its lexicon, which is facilitated through language contact (Nair, 2008). The phenomenon of code-switching and lexical replacement has a very little attention in the domain of psycholinguistics research (Adler, 2006; Tamaoka, and Miyaoka, 2003). An attempt is made here to describe the psycholinguistics perspective of code-switching and lexical replacement mechanisms in Odia, as an Indo-Aryan language, spoken in the Indian territory of Odisha. Odia has 
been adapted many words from other languages (as seen in figure.1) in the due course of borrowing and lexical enrichment (Dalai, 2004; Mohanty, 1986)

English donates many words to Odia in a contact situation. Odia bi-multilingual speakers have a tendency to use English borrowed words while speaking and writing of their mother tongue irrespective of their education and medium of instruction (Dalai, 2004; Annamalai, 2004). This adaptation process may replace old Sanskrit tatsam words (STWs) and the use of English borrowed words (EBWs) in Odia. We know very little about the psycholinguistic perspectives on how the native speakers encode, store and retrieve orthographic representation of English borrowed words and their semantic equivalent Sanskrit tatsam words in Odia.

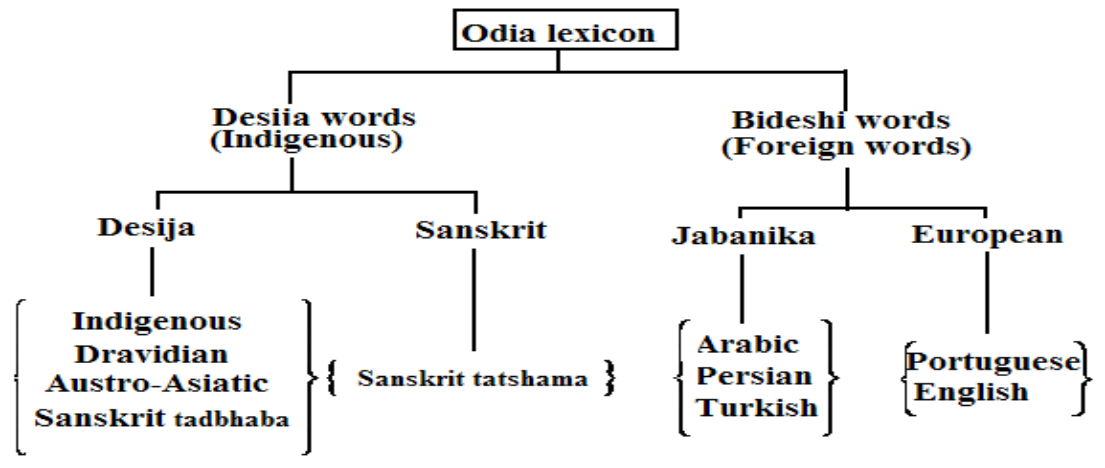

Figure.1 Stratification of Odia words based on the source languages

\section{The current study}

The aim of the current study is to investigate whether the native speakers prefer to use English borrowed words or equivalent Sanskrit tatsam words in a competing situation of language contact. Do the Odia bi-multilingual speakers process English borrowed words differently as compared to equivalent Sanskrit tatsam words? Do bimultilingual Odia speakers process old Sanskrit tatsam words much better and faster than new English borrowed words in Odia? Is there any role of syllabic length of target STWs and EBWs in the process of adaptation or replacement of STWs and EBWs? Does the minimal disyllabic structure of Odia play any role in the process of adaptation or replacement of STWs and EBWs? Answer to these fundamental questions can provide mechanisms of lexical switching and replacement between Odia and English. Moreover, the comprehension of orthographic representation of target English borrowed words and Sanskrit tatsam embedded with the preceding Odia (desija) and English recent prime words may provide a cross-talk between code-switching and replacement of words in Odia. Thus, the current research presents an investigation on how a decrease in perception determines the replacement of underuse old Sanskrit tatsam words (STWs), and the increase of perception manifests the adaptation of overuse new English borrowed words (EBWs). A relationship between lexical replacement and code-switching has been established by examining the perceptual mechanisms of lexical adaptation in Odia. To test adaptation of new English words in terms of perceptual rate, a psycholinguistic experiment on old Sanskrit tatsam $(n=60)$ and their semantic-equivalent English-borrowed words $(n=60)$ was conducted by using semantic-priming paradigm.

\section{Methods}

The aim of the current study is to investigate whether Odia-English bilingual speakers' process target Sanskrit tatsam words (STWs) differently than their equivalent English borrowed words in Odia. Participants may prefer to use English borrowed words (EBWs) in the place of Sanskrit tatsam words in Odia. 


\section{Participants}

Participants were 14 native Odia right-handed speakers studying at the National Institute of Technology, Raipur, India (3 females, age $M=19.9$ years, range= years $S D=1.6$ ). Though their mother tongue was Odia, but they studied English as a subject from $4^{\text {th }}$ class-onwards. All Odia-English bilingual-participants who were healthy, and had no normal or corrected vision, gave their written consent to voluntarily participate in the experiment.

\section{Stimuli}

Target stimuli were old Sanskrit disyllabic $(n=33)$ and tri-syllabic $(n=27)$ tatsam words $(n=60)$, and their equivalent English monosyllabic $(n=15)$, disyllabic $(n=25)$ and tri-syllabic $(n=20)$ borrowed words $(n=60)$ in Odia. In addition, priming words comprising negative and positive Odia native (ON) (i.e., desija, n=120) and recent (nonce) English words (REW) $(n=120)$ were selected based on their positive and negative association with target Sanskrit tatsam and English borrowed words. Both target and prime words were presented in Odia orthography following their correct presentation in Odia dictionaries (Padhi,1988; Pradhan, 1997/2004). Odia, as an Eastern variety of Indo-Aryan family of language, is spoken in the territory of Odisha, India (Mohanty, 1986). Orthographic representations of word stimuli were evaluated by native Odia speakers by using a value judgment task following a 7-point Likert scale, representing 1-7 extreme-to-less degree foreignness (Englishnization) of borrowed words. Ten participants were asked to judge the degree of foreignness while reading English borrowed words and equivalent native words. The middle number 4 represented neutral words. We excluded neutral words from the experiment. High- rating English borrowed words $(\mathrm{N}=75)$ were selected to design the target stimuli for the experiment. Similarly, the target Sanskrit tatsam words were evaluated by Odia speakers considering their old and new form of nativization status in Odia. High rate of old Sanskrit tatsam words were included in the experiment. STWs were borrowed from Sanskrit and maintained similar forms in writing as in donor language. Syllabic length of the target words were controlled and kept maximum three (trisyllabic) syllables to maintain homogeneity in word length and to examine any processing effect arising due to differences in the number of syllables in target words. All words were written in black Odia letters by keeping the font size at 28. Target Sanskrit tatasam and English borrowed words were matched either negatively or positively with the preceding Odia and English prime words, as the following examples show in table 1.

Table 1 shows the Sanskrit tatsam and English borrowed words with their prime words establishing positive and negative meaning.

\begin{tabular}{|c|c|c|c|c|c|}
\hline \multicolumn{4}{|c|}{ Prime words } & \multicolumn{2}{|l|}{ Target words } \\
\hline \multicolumn{2}{|l|}{ Odia words } & \multicolumn{2}{|c|}{ English words } & Sanskrit (Tatsam) & English borrowed \\
\hline Positive & Negative & Positive & Negative & & \\
\hline $\begin{array}{l}\text { ctpe } \\
/ \mu \mathrm{O} v \delta \rho \mathrm{O} /\end{array}$ & $\begin{array}{l}\text { chĐZP } \\
/ \mu \mathrm{O} v \delta \mathrm{I} \rho /\end{array}$ & $\begin{array}{l}\text { 669qm? } \\
/ \tau \varepsilon \mu \pi \nu \lambda /\end{array}$ & $\begin{array}{l}\text { Фิ6Я66"। } \\
/ \kappa \rho 1 \mu \varepsilon \tau о \rho 1 /\end{array}$ & /ıø. $\omega O \rho /$ & $/ \gamma \mathrm{O} . \pm \mathrm{O} /$ \\
\hline $\mathrm{SP}[\mathrm{PaO}$ & kĐePaĐ & $\mathrm{DB}_{\_} c$ & m@jç & Att & $k D^{\prime} p$ \\
\hline
\end{tabular}




\begin{tabular}{|l|l|l|l|l|l|}
\hline$/ \varphi \mathrm{I} \tau \mathrm{I} \beta \alpha /$ & $/ \eta \alpha \rho \mathrm{I} \beta \alpha /$ & $/ \mathrm{v} \mathrm{v} /$ & $/ \lambda \mathrm{O}: \sigma /$ & $/ \alpha . v \mathrm{O} v . \delta \mathrm{O} /$ & $/ \eta \alpha \pi \mathrm{r} /$ \\
\hline
\end{tabular}

Two sets of stimuli based on target Sanskrit and English borrowed words were formed and equally divided into positive $(n=240)$ and negative $(n=240)$ trails by using semantic priming paradigm. The meaning of priming word is to facilitate the processing of target words' meaning.

\section{Procedures}

Participants were seated in a dark room, in a sound-attenuated chamber, facing an LCD screen at a distance of approximately $90 \mathrm{~cm}$. Following the orthographic representation of Odia, target Sanskrit tatsam and English borrowed words were presented one by one after the positive or negative priming words (both English and Odia) on a white-17-inch LCD screen. Word stimuli were presented using 'E-prime' software (3.0 version). They were instructed that words would appear one after another on the screen to make a complete negative or positive associative meaning between first and second word. Both prime and target words appeared for a duration of 500 ms each with an inter-stimuli interval of $250 \mathrm{~ms}$ (Fig.1). The duration gap between prime and target word was kept 400ms. All trails $(n=480)$ were randomized within four blocks to avoid any sort of regular response pattern. Each block contained the equal number $(n=120)$ of trails. First block contained target Sanskrit tatsam which were preceded by positive-negative Odia (desija) and English (nonce) priming words, whereas the second block comprised target English borrowed words that were preceded by same priming words. Third block contained target Sanskrit tatsam and English borrowed words that were preceded by positive-negative prime English (nonce) words, whereas the fourth block comprised the same target words preceded by positive-negative Odia (desija) words. A blank white screen appeared at the end of each trail as a hint to participants to perform their task, as seen in the figure 2 .

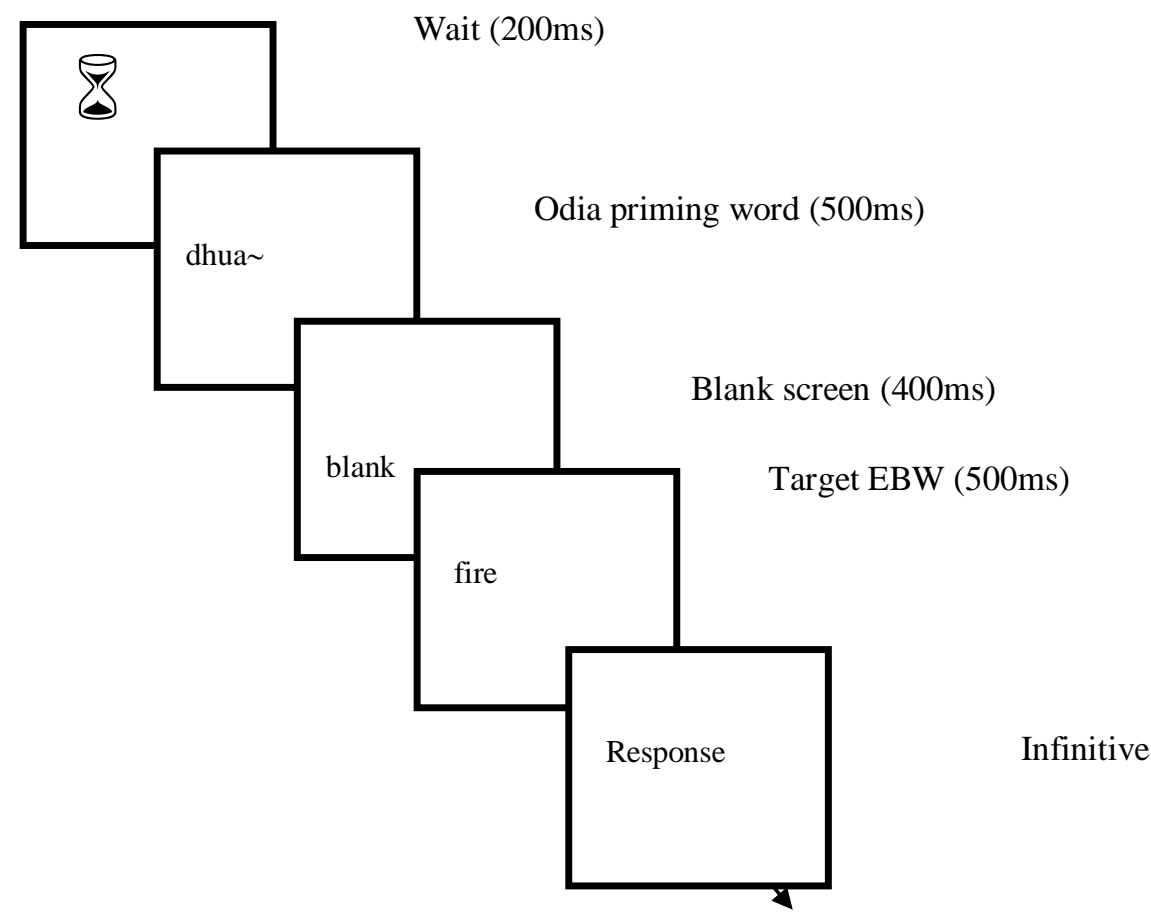

Figure 2 shows a schematic diagram of sequence of words in a trail. The Odia (disija) priming word 'dhua ' with target English borrowed word 'fire' establishes positive priming association. 
Participants were instructed to read both words in a single trail, and comprehend the meaning to assess the positive and negative semantic relatedness between prime and target words. They were asked to respond by pressing left arrow-key for positive and right arrow-key for negative meaning irrespective of the presence and absence of Sanskrit tatsam and their equivalent semantic English borrowed words. They were not aware about the presence of two different sets of target words which are semantic competitors in Odia. Response time was kept infinite. However, participants were asked to response as early as possible. A practice session consisting of 10 trails was given to each participant to familiarize them with the task and response. No trail was repeated in this experiment.

\section{Behavioural data analysis}

Behavioural reaction time (ms) was collected from each participant across blocks within trials. Average reaction time and accuracy were measured for each participant using the Microsoft excel. Further, a series of $(2 \times 2 \times 3)$ ANOVA was conducted to observe the main and interaction effect between experimental variables such as 2 target words (STW, EBW), 2 primping (Negative, Positive for both Odia and English), and 3 length of words (monosyllabic, disyllabic, tri-syllabic). The program and packages used to conduct the repeated measure ANOVA analysis was the SPSS statistics software considering dependent variables that were gathered throughout the experimental phase. These variables will be statistically described more closely in the result section.

\section{Results}

Mean RT (ms) values were presented for a repeated-measure ANOVA, using 2 priming (positive, negative) $\times 2$ language priming (Odia priming, English priming) $\times 2$ Sanskrit tatsam words (disyllabic, tri-syllabic). In this case, the target words were disyllabic and tri-syllabic Sanskrit tatsam words that were preceded by priming Odia (desija) and English recent borrowed words. As expected, participants were responded faster to the positive priming (P-priming) than negative priming (N-priming) words, revealing a significant, $F(1,13)=4.91, p=.04$, $\eta^{2} p=.53$, main effect of priming, as shown in the figure 3 . Considering the minimal disyllabic structures of Odia, the main effect of syllabic structures of Sanskrit tatsam words was significant, $F(1,13)=12.97, p=.003$, $\eta^{2} p=.91$, suggesting a quicker response for disyllabic than tri-syllabic Sanskrit tatsam words (STWs), as indicated in the figure 4. The ANOVA also yielded a significant, $F(1,13)=16.18, p=.001, \eta^{2} p=.96$, interaction effect between semantic priming-words (positive, negative) and priming language words (Odia, English) revealing a quicker response time for positive English priming than negative English priming words. Post-hoc test using Tukey procedures confirmed significant differences, $(t(14)=5.88, \mathrm{p}=.005)$, between positive and negative priming in which positive English priming words (ENG) were processed faster than negative English priming words, as depicted in the figure 5. In this test, the interaction between language related priming words (English, Odia) and target Sanskrit words (disyllabic, tri-syllabic) was close to significant, $F(1,13)=$ $3.20, p=.09, \eta^{2} p=.38$, in which participants responded faster to disyllabic Sanskrit tatsam than tri-syllabic Sanskrit tatsam words when both these target words were preceded by English priming words. In post-hoc analysis, the mean comparisons showed that English priming words significantly, $(t(14)=4.93, \mathrm{p}=.01)$, enhanced the processing of STWs which was faster for disyllabic than tri-syllabic STWs. Notably, a three-way cross-interaction of dependant variables: priming (positive, negative), language priming (English, Odia) and Sanskrit tatsam words (disyllabic, tri-syllabic) was significant, $F(1,13)=5.58, p=.03, \eta^{2} p=.58$, revealing a an interference of negative and positive English priming words on target Sanskrit disyllabic and tri-syllabic tatsam words, as shown in the figure 5. Post-hoc analysis showed a contrastive mean values between disyllabic and trisyllabic STWs, which was significantly, $(t(14)=8.53$, p=.0008), differed indicting faster response for disyllabic STWs than tri-syllabic STWs when these target words were embedded with previous negative English primingwords. Similarly, positive English priming-words enhanced the processing of tri-syllabic STWs more rapidly than negative priming words $(t(14)=6.68, \mathrm{p}=.006)$. Interestingly, these interaction effects indicated that 
negative semantic-associations of English priming-words heighten the rapid processing disyllabic STWs, whereas positive semantic association of English priming-words facilitated the response time of tri-syllabic STWs. Taken together, these findings partially support our hypothesis there is a greater degree of codeswitching from English priming words to target Sanskrit tatsam words that enhance the processing of target Sanskrit words.

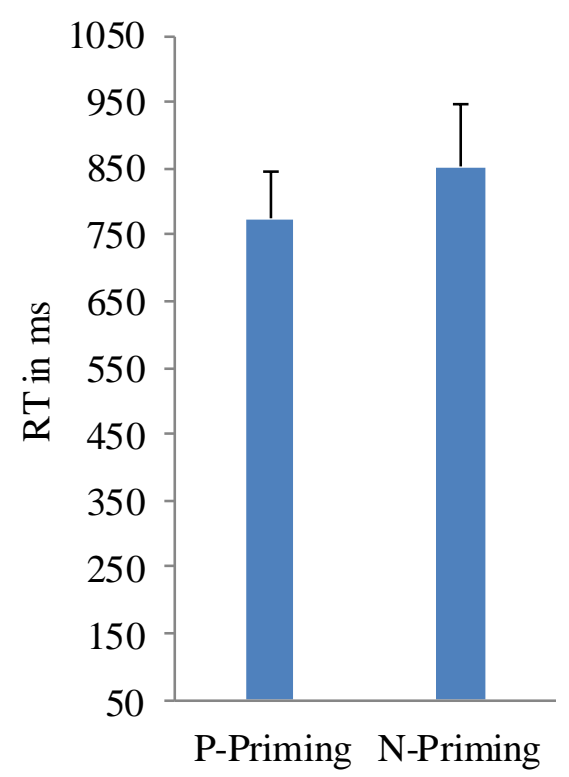

Figure 3 shows the main effect of priming.

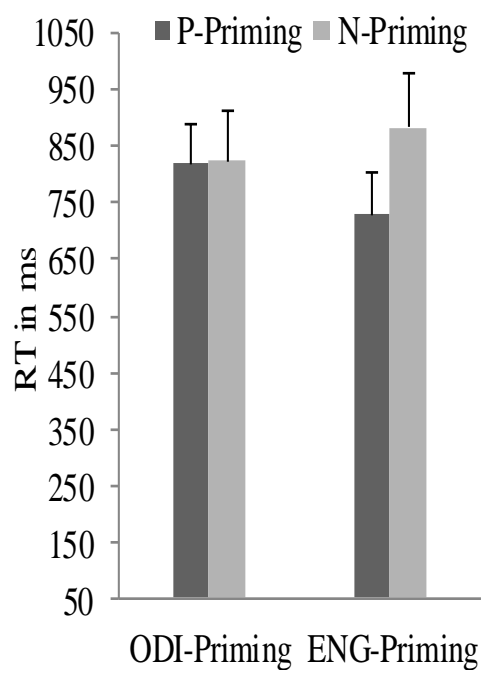

Figure 5 shows the differences between English on STWs.

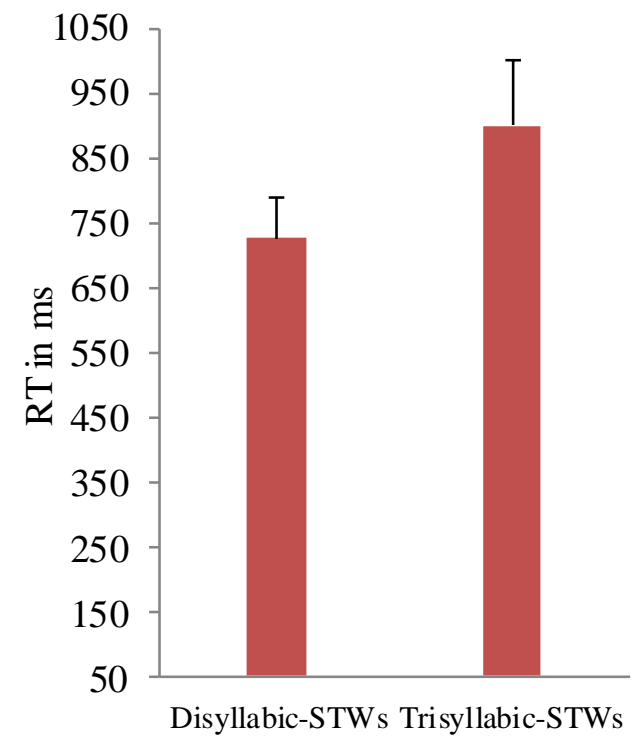

Figure 4 shows the effect of syllabic length and STWs.

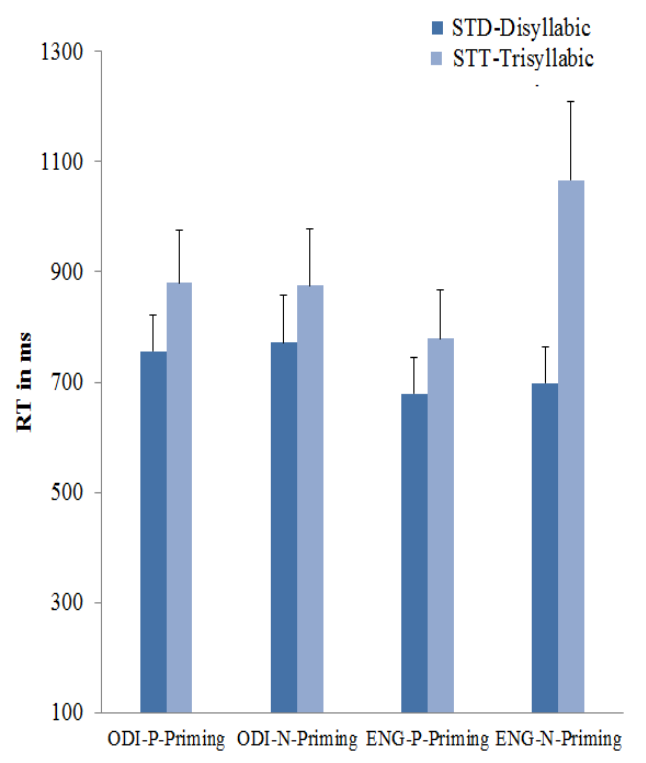

Figure 6. shows the interference of English priming words.

In addition to these interference of priming with target STWs, the analysis of RTs (ms) was also conducted targeting the interaction effect of the same priming (positive and negative Odia and English) words on target English (mono-, di-, and tri-syllabic) words. A three-way repeated measure ANOVA was performed by means of 2 priming (positive, negative) $\times 2$ language priming (Odia priming, English priming) $\times 3$ English borrowed (mono-syllabic disyllabic, tri-syllabic) words. The ANOVA identified a significant main effect of priming words of language (Odia, English), $F(1,13)=5.58, p=.03, \eta^{2} p=.58$, suggesting a rapider response to Odia than English priming words, as shown in figure 6. Follow-up analyses showed that the main effect of target 
English borrowed words (EBWs) was significant revealing quicker response for mono- and disyllabic length of words than tri-syllabic words, $F(1,13)=7.30, p=.008, \eta^{2} p=.86$, as depicted in the figure 7 . Considering the minimal structures of disyllabic words in Odia, participants might have mapped English monosyllabic and disyllabic borrowed words together with the schema of disyllabic word structures, thereby facilitating the processing of similar target EBWs. The processing of these target EBWs were significantly influenced by the preceding Odia and English priming words. A two way interaction effect between language priming (Odia, English) and target EBWs (Mono, di-, tri-syllabic) was significant, $F(1,13)=7.86, p=.007, \eta^{2} p=.88$, suggesting faster response time for monosyllabic EBWs with preceding Odia priming than English priming words, as shown in figure 8. Odia (desija) priming words influenced the monosyllabic EBWs because these monosyllabic borrowed words were adapted as disyllabic words in Odia. Post-hoc analysis identified a trend showing that mean differences, $(t(14)=5.25, \mathrm{p}=.02)$, between response times of target monosyllabic EBWs with preceding Odia and English priming words, suggesting faster reaction time for monosyllabic EBWs embedded with Odia than English priming words. To sum up, Odia priming words are associated with target monosyllabic English borrowed words to enhance the adaptation of monosyllabic English words as disyllabic words, whereas English priming words are surrounded with disyllabic Sanskrit tatsam words to preserve the disyllabic structures of Odia words.
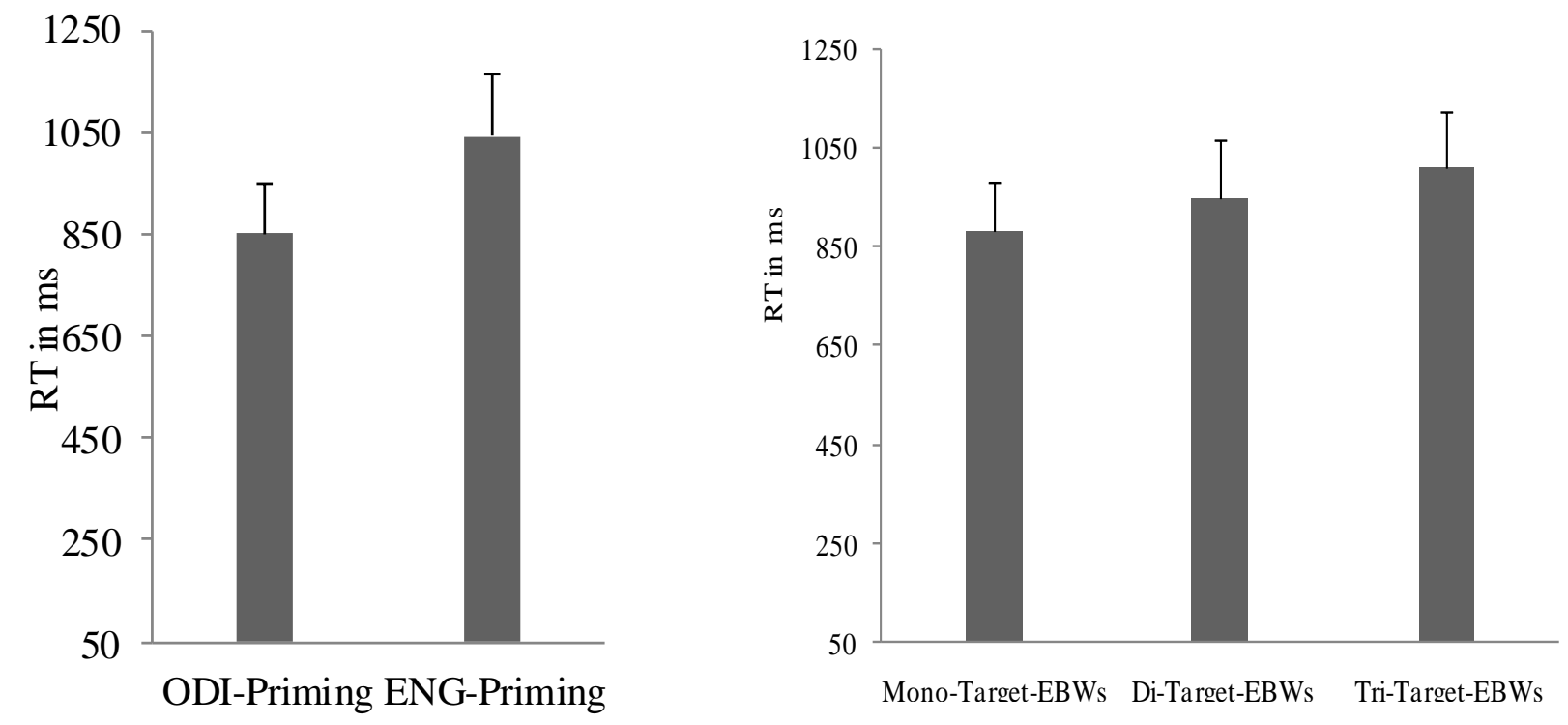

Figure 7 shows the fast response to Odia priming words. Figure 8 shows the effect of syllabic length of EBWs.

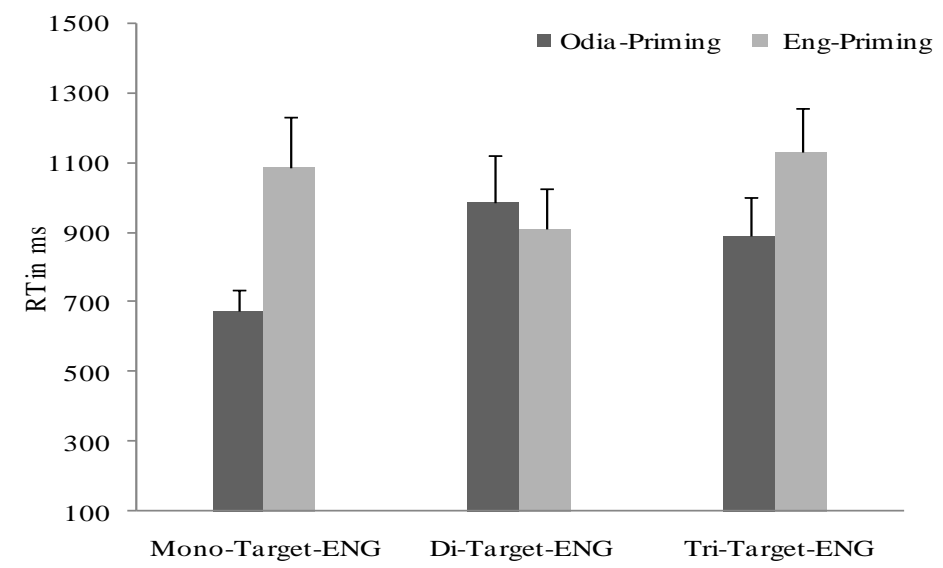

Figure 9 shows the quicker response to mono-syllabic target words of English 
Further, the mean RTs (ms) of target disyllabic and tri-syllabic Sanskrit tatsam and English borrowed words were analysed with respect to the preceding positive and negative English nonce words. Do the preceding English priming words have any impact on the processing of following target di-trisyllabic Sanskrit tatsam and English borrowed words? A three-way ANOVA, was performed using of 2 English priming (positive, negative) words $\times 2$ syllabic length of target (disyllabic, tri-syllabic) words $\times 2$ target borrowed (STWs, EBWs) words. The ANOVA identified a significant, $F(1,13)=31.37, p=.001, \eta^{2} p=.99$, main-effect of priming indicating faster response time for negative than positive English priming words. This finding confirms that the negative English borrowed words are adapted much faster than positive English words, as shown in the figure 9. It was also found that the main effect of syllabic length (di- and tri-syllabic) of target words was significant, $F(1,13)$ $=22.63, p=.001, \eta^{2} p=.99$, indicating quicker response for disyllabic than tri-syllabic length of target words, as indicated in figure 10. This effect of syllabic length of target words interfered with the target words of language (Odia, English), as confirmed by a significant, $F(1,13)=5.64, p=.03, \eta^{2} p=.59$, two-way interaction effect between 2 syllabic length (disyllabic, tri-syllabic) of target words $\times 2$ target language (STWs, EBWs) words, indicating faster response for disyllabic than tri-syllabic only for EBWs, but not for STWs, as shown in the figure 11. Post-hoc test recognised a significant reaction time, $(t(14)=5.25, \mathrm{p}=.01)$, which was quicker only for disyllabic than tri-syllabic EBWs.

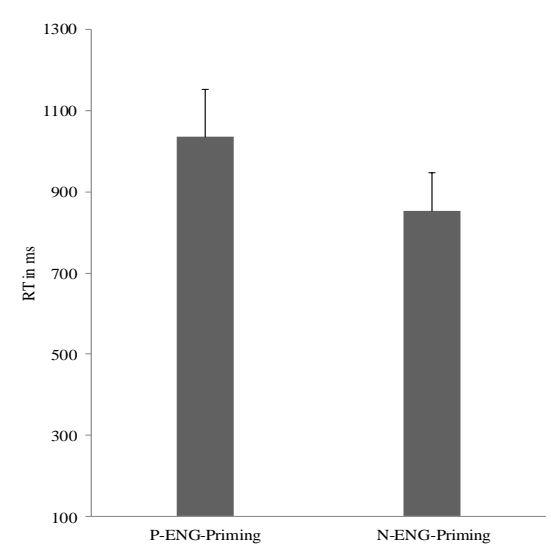

Figure 9 shows the quick response to negative ENG priming.

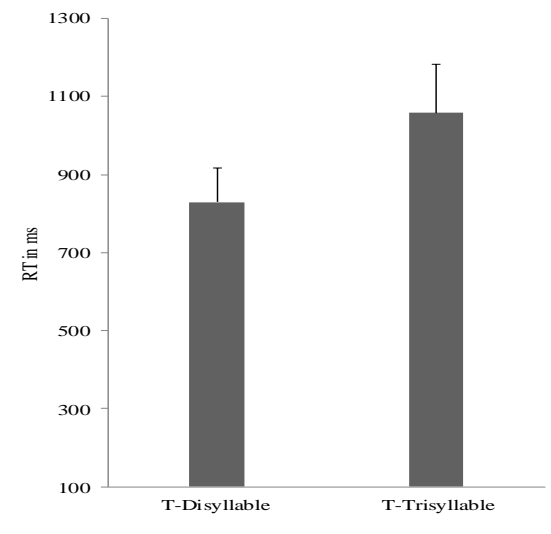

Figure 10 shows the quick response to target disyllabic.

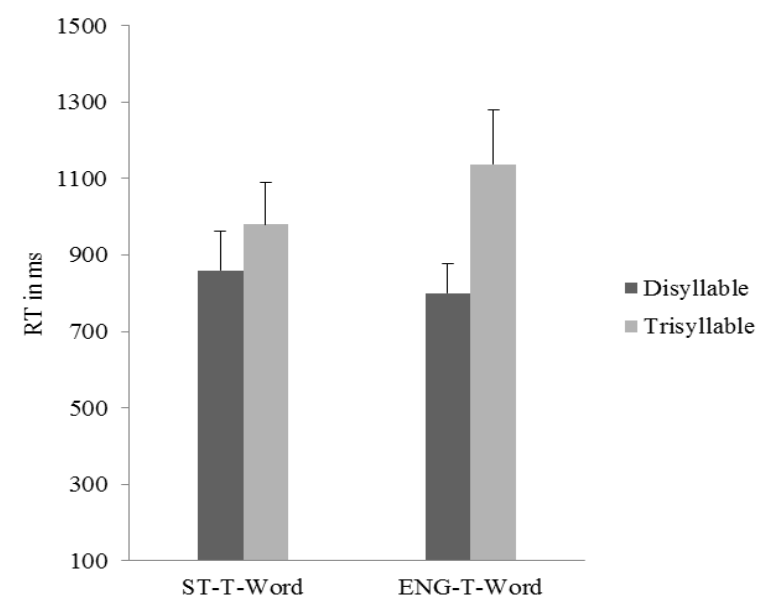

Figure 11 shows the faster reaction time for disyllabic English than trisyllabic words 
Overall, these findings suggest that disyllabic target EBWs are processed faster than tri-syllabic words confirming the minimal disyllabic structures of Odia (Das, 2006; Vijayakrishnan, 2000). Negative English prime words are processed much faster than positive- English prime words. However, in the case of Odia (desija) priming words, positive-priming words were processed faster than negative priming words, indicating a significant, $F(1,13)=10.36, p=.007, \eta^{2} p=.84$, main effect of Odia positive and negative priming words.

\section{Discussion and conclusion}

To sum up, this result shows the main effect of priming in which positive priming words are processed much easier than negative priming words. This finding clearly confirms that positive aspects of language or emotion are processed much earlier than the negative aspects of language. It is also observed that positive English priming, but not the negative English priming words, were processed faster than Odia priming words. English priming words dominates the Odia priming words in terms of processing. Therefore, participants takes more time to respond the negative priming words when these words are associated with target Sanskrit tatsam words. These effects clearly suggest that negative priming words are not associated with target Sanskrit tatsam words. When native bilingual speakers of Odia process Old Sanskrit words, they may need the help of positive priming words that enhance the comprehensibility of Old words. Since old words are in permanent and remote memory of the native speakers, they need to positive priming words to facilitate the decoding process. Further, it is observed that syllabic length of words is interfered with the processing target STWs, in which disyllabic STWs were processed faster than tri-syllabic words, confirming the minimal disyllabic structure of Odia. Disyllabic words are processed faster than tri-syllabic words Odia. This fact is also found in the case of target English words, in which mono and disyllabic target EBWs are processed faster than tri-syllabic target EBWs. Negative English priming words enhance the processing of target disyllabic STWs, whereas positive English priming words facilitate the processing of tri-syllabic STWs. Overall, English priming words enhance the processing of STWs. All these finding clearly define the psycholinguistic nature of language in a contact situation (Peperkamp, 2004).

This current research shows that the decrease in perception determines the replacement of underuse old Sanskrit tatsam words, and the increase of perception manifests the adaptation of overuse English borrowed words. Further, this study opens a new path to observe the psycholinguistic aspects of language enrichment and birth through code-mixing, code-switching and lexical borrowing (Peperkamp, et al. 2008). The degeneration of a language largely happens due to old word replacement and shift, which may lead to language death. In this context, code-switching from Odia to English looks suitable and does not encounter any tension on the part of the audience. Taken together, the results of the present study confirm the hypothesis that old Sanskrit words have a tendency to be replaced by English disyllabic words. In other words, disyllabic English words are adapted much faster than tri-syllabic words in the place of underuse old Sanskrit tatsam words. If old words do not have flexible functions or use in communication according to the need and demand of speakers, there will be no options for speakers to preserve old words. Speakers often replace non-functional or defunct word with functional one.

\section{References}

Adler, A.N., 2006. Faithfulness and perception in loanword adaptation: A case study from Hawaiian. Lingua, 116(7), pp.1024-1045.

Annamalai, E., 2004. Nativization of English in India and its effect on multilingualism. Journal of Language and Politics, 3(1), pp.151-162.

Calabrese, A. and Wetzels, W.L. eds., 2009. Loan phonology (Vol. 307). John Benjamins Publishing.

Cannon, G. and Egle, B.M., 1979. New borrowings in English. American Speech, 54(1), pp.23-37.

Chaudhary, S., 2009. Foreigners and foreign languages in India: A sociolinguistic history. Cambridge India. 
Clyne, M.G., 2003. Dynamics of language contact: English and immigrant languages. Cambridge University Press.

Dalai, U.P., 2004. Language modernization in Oriya newspapers. IJDL. International journal of Dravidian linguistics, 33(2), pp.105-124.

Das, R.K., 2006. Role of SSP in syllabification of words in Oriya. IJDL. International journal of Dravidian linguistics, 35(1), pp.97-110.

Haspelmath, M., 2008. Loanword typology: Steps toward a systematic cross-linguistic study of lexical borrowability. Empirical Approaches to Language Typology, 35, p.43.

GOPINATHAN NAIR, B., 2008. DIALECT SURVEY OF MALAYALAM IN RETROSPECT. IJDL. International journal of Dravidian linguistics, 37(1), pp.169-190.

Padhi, B. B., 1998. Oriya bhashara roopatattwa. New Students’ Publishers, Cuttack.

Paradis, C. and LaCharité, D., 1997. Preservation and minimality in loanword adaptation. Journal of linguistics, 33(2), pp.379-430.

Paradis, M., 1993. Linguistic, psycholinguistic, and neurolinguistic aspects of" interference" in bilingual speakers: The activation threshold hypothesis. International Journal of Psycholinguistics.

Patnaik, B. N. and I. Pandit., 1986. Englishnization of Oriya. pp. 232-243. InB. H. Krishnamurti, C. P. Masica, andA. K. Sinha (eds.) Modernization of South Indian languages: Lexical Innovations in Newspaper Languages. MotilalBanarsidass Publishers, Delhi,

Peperkamp, S., 2004, June. A psycholinguistic theory of loanword adaptations. In Annual Meeting of the Berkeley Linguistics Society (Vol. 30, No. 1, pp. 341-352).

Peperkamp, S., Vendelin, I. and Nakamura, K., 2008. On the perceptual origin of loanword adaptations: experimental evidence from Japanese. Phonology, 25(1), pp.129-164.

Pradhan P. 1997/ 2004. BhashashastraParichaya. State Bureau of Text, Odisha.

Romaine, S., 2006. Bilingualism. Blackwell Publishing, USA.

Tamaoka, K. and Miyaoka, Y., 2003. The cognitive processing of Japanese loanwords in katakana. Japanese Psychological Research, 45(2), pp.69-79.

Thomson, S. G. Language Contact. Wahington, D.C.Georgetown University Press, 2001.

Thomson, S.G. and Kaufman, T., 2004. Language Contact. Georgetown University Press, Wahington, D.C.

Grey, T.S. and Kaufman, T., 1988. Language contact, creolization, and genetic linguistics. Berkeley.

Vendelin, I. and Peperkamp, S., 2006. The influence of orthography on loanword adaptations. Lingua, 116(7), pp.996-1007.

Vijayakrishnan, K.G., 2007. The Disyllabic Word Minimum: Variations on a Theme in Bangla, Punjabi and Tamil. Linguistic Theory and South Asian Languages: Essays in Honour of KA Jayaseelan, 102, p.237. 